\title{
Communicating with Mechanically Ventilated Patients Who Are Awake. A Qualitative Study on the Experience of Critical Care Nurses in Cyprus during the COVID-19 Pandemic.
}

Maria Kyranou ( $\nabla$ maria.kyranou@cut.ac.cy)

Cyprus University of Technology https://orcid.org/0000-0002-8724-2429

Charikleia Cheta

Cyprus University of Technology: Technologiko Panepistemio Kyprou

Eliada Pampoulou

Cyprus University of Technology: Technologiko Panepistemio Kyprou

\section{Research}

Keywords: Intensive Care Unit, Communication, Sedated, Conscious, Awake, Mechanically Ventilated Patients, Nursing, Qualitative research.

Posted Date: July 13th, 2021

DOI: https://doi.org/10.21203/rs.3.rs-667143/v1

License: @ (i) This work is licensed under a Creative Commons Attribution 4.0 International License. Read Full License 


\section{Abstract}

Background: Modern protocols for light sedation in combination with the increased turnover of COVID-19 infected patients hospitalized in Intensive Care Units (ICUs) have increased the number of patients who are mechanically ventilated and awake. Nurses require specific skills to care for this vulnerable group of patients. At the same time, nurses report feeling inadequate and frustrated when they attempt to establish communication with mechanically ventilated, conscious patients.

Methods: The purpose of this study was to explore nurses' experiences taking care of conscious, intubated patients in the intensive care unit. The research questions aimed to identify $\mathrm{i}$. the strategies that nurses use for communicating with patients, and ii. the barriers in communication between nurses and patients. This study employed a qualitative design. Data were collected using semi-structured interviews with 14 intensive care nurses working at ICUs in four different hospitals of Cyprus. The data were analyzed by applying thematic analysis.

Results: We identified several strategies of unaided (movements -lips, hands, legs- facial expressions, gestures, touching) and aided forms of communication (pen and paper, boards, tablets, mobiles) used by nurses to communicate with patients. Additionally, barriers in communication were reported by participating nurses mainly pertaining to patients', nurses' characteristics and the ICU environment. The health protocols imposed by the pandemic added more barriers in the communication between nurses and patients mostly related to the use of protective health equipment.

Conclusions: The results of this study, combined with those of others in different countries, point to the difficulties nurses face when trying to communicate with conscious patients during mechanical ventilation. It appears that the complex communication needs of this group of patients are not being met mainly due to the lack of nurses' training and of appropriate equipment to facilitate alternative and augmentative communication. Our study is amongst the first to add that the protective health protocols due to the pandemic imposed further communication barriers. Undoubtedly, the recognition of such an important issue creates an urgent need to educate nurses in alternative ways of communication with mechanically ventilated, conscious patients during their ICU stay.

\section{Background}

Never before did mechanical ventilation concern the public as much as during the past year due to mass admissions and turnover of patients infected by COVID-19 in Intensive Care Units (ICUs). Undoubtedly, patients' needs after such an invasive medical procedure are numerous and many studies have explored them previously (1). This study is not about these needs but about the way to express them to health care professionals. In other words, when intubated patients slowly regain their consciousness, are they able to communicate to physicians and nurses what they need? Put differently, are health care professionals able to approach and understand this vulnerable group of patients to ensure that they are not left abandoned and conscious in the threatening ICU environment?

In combination with the recent pandemic, modern protocols for light sedation have led to the increase in the number of patients that are in mechanical ventilation but are able to communicate. Guidelines from the Society of Critical Care Medicine, published in 2018, determined that targeted light sedation for adult patients is the ideal level of sedation for treating agitation and pain in the mechanically ventilated patient in the ICU (Devlin et al, 2018). Furthermore, the use of targeted light sedation is associated with a reduced rate of tracheostomy placement, shorter time to extubation (2), decreased ICU length of stay, hospital mortality (3), memory problems and symptoms of posttraumatic stress disorder related to ICU admission (4). Inevitably, these practices increase the number of patients who are awake and potentially able to communicate while mechanically ventilated.

Despite the benefits of light sedation during mechanical ventilation patients experience important limitations in their ability to communicate their needs. Previous studies found that patients experience pain and feelings of panic when they are awake during respiratory treatment (5). Additionally, communication problems due to endotracheal intubation are associated with severe stress and emotional reactions among ICU patients $(6,7)$. Often, critical care professionals focus in the assessment of life-threatening signs and consider communication as a luxury in their time-limited environment (8-10). Furthermore, their lack of education on alternative ways of communication with special patient groups (11) frequently leads to mistaken interpretation of patients' signals and subsequently to failures in their care (12).

Communication is a fundamental human right (United Nations, 1948) and needs to be ensured even for people with disabilities or temporary impairments (Article 21 of the Convention on the Rights of Persons with Disabilities, United Nations, 2006) (13). Previous studies demonstrated that the characteristics of the ICU environment, patients' physical and emotional status as well as lack of training in alternative communication strategies are some of the reasons why nurses fail to understand patients hospitalized in an ICU, who are mechanically ventilated but conscious (8-10, 14-21). Nevertheless, most of these studies took place in northern Europe, the United States and Australia whereas no study investigated this problem In Mediterranean countries. Given that different professional standards apply for critical care nursing practice among countries it is imperative to investigate whether nurses face similar problems in places of the world where understaffing is a major problem and advanced nursing practice has not been established yet. Hopefully, such an effort with draw attention to the problem and might lead to the adoption of solutions to close the gap between theory and practice for the ideal care of intubated, conscious ICU patients.

\section{Theoretical Framework}

Communication is integral for the creation of a trusting, therapeutic relationship between nurses and patients. For patients hospitalized in an ICU, in particular, the quality of patient communication with health care professionals associates with their agony for survival (22). The theoretical framework of this study is informed by traditional nursing theories on communication (23) and encompasses elements from the dyadic interpersonal communication model (24) which acknowledges feedback of nurses as a critical element for the accuracy of verbal and non-verbal communication. Furthermore, the hypotheses underlying the study design stem from the recognition that patients who are mechanically ventilated and awake in an ICU have complex communication needs (25). As a result, augmentative and alternative communication strategies to address their needs are required.

Page $2 / 12$ 
Augmentative and alternative communication (AAC) is a clinical field that addresses the needs of individuals with complex communication disorders characterized by impairments in speech-language production and/or comprehension, including spoken and written modes of communication (26). AAC uses a variety of techniques and tools, including picture communication boards, line drawings, speech-generating devices, tangible objects, manual signs, gestures, and finger spelling, to help the individual express thoughts, wants and needs, feelings, and ideas. AAC is augmentative when used to supplement existing speech, and alternative when used in place of speech that is absent or not functional. AAC may be permanent, as when used by an individual who will require the use of some form of AAC throughout his or her lifetime or temporary, as when used by patients postoperatively or while intubated in critical care settings (26). AAC is typically divided into two broad categories-unaided and aided. Unaided forms of AAC do not require an external tool, only some degree of motor control (ASHA). Aided forms of AAC require some form of external support-either electronic or nonelectronic.

ACC was shown to be useful when used by clinicians and ICU patients with temporary disability to improve communication (27, 28). For the scope of this study we adopted the description of disability by the International Classification of Functioning, Disability and Health (ICF) as an interaction between features of the person and features of the person's environment (World Health Organization [WHO], 2013)(29). The three levels of functioning according to ICF -body function and structure, activity and participation- interact with aspects of the AAC system (unaided or aided) in determining the function of a person (30). Furthermore, the Participation Model is directly related to the purposes of this study as it provides a framework for assessment and effective AAC communication between the sender (e.g. patient) and the receiver (e.g. nurse). It takes into consideration the environment within which communication takes place, existing practices as well as the communication partners' (e.g. nurses) attitudes, knowledge and skills (26).

\section{Methods}

\section{Design}

This study used a qualitative descriptive design (31) and adapted the Standards for Reporting Qualitative Research checklist (32).

\section{Research Purpose and Aims}

The purpose was to explore nurses' experiences while taking care of conscious, intubated patients in the Intensive Care Unit. Specifically, the research aims focused to identify:

1. the strategies that nurses use to communicate with patients when they are mechanically ventilated but awake,

2. the barriers in the communication between nurses and patients.

\section{Setting and Participants}

The participants consisted of 14 nurses working at ICUs in four different hospitals of Cyprus who were purposively identified and selected (33). Since the aim was to select in-depth information about lived experiences, a sample of 14 nurses was deemed adequate (34). The inclusion criteria were: i) working in an ICU, and ii. waving worked before with people who were mechanically ventilated and awake in an ICU. The exclusion criteria were: i. nurses working in paediatric ICUs or neonatal ICUs, and ii. nurses not willing to participate. As shown in Table 1, four of the nurses were female and ten were male. The nurses' age ranged between 28 and 45 years (mean age $=34.5$ years). Their years of nursing experience ranged between 2 and 24 years (mean = 11.5 years), and of ICU clinical experience between $1-15$ years (mean $=5.5$ years).

Table 1

Characteristics of the nurses

\begin{tabular}{|ll|}
\hline \multicolumn{2}{|c|}{ Characteristics of the nurses } \\
\hline Sex & \\
\hline Female & $\mathbf{1 4})$ \\
\hline Male & 4 \\
\hline Years of Nursing Experience & 10 \\
\hline Mean/median & $11.5 / 11.25$ \\
\hline Range & $2-24$ \\
\hline Years of Intensive Care Nursing Experience \\
\hline Mean/median & $5.5 / 5.5$ \\
\hline Range & $1-14$ \\
\hline
\end{tabular}

\section{Data Collection}

Semi-structured interviews were used for data collection. The interviews were based on an aide-mémoire that was developed based on the research questions of the study. The main questions asked were: 1) Please describe your experience when attempting to communicate with a patient who is ventilated and conscious, 2) What strategies to do you use in order to communicate with this group of patients, 3) What barriers do you experience in your attempt to communicate, 4) In the ICU you are working do you have any communication tools that you can use to facilitate communication with these patients, 5) Would you like to receive training on the different strategies that could be utilized for communicating efficiently with patients who are ventilated and conscious? 
Data collection took place between September 2020 and May 2021. Interviews were held in physical presence for 8 participants and online for 6 participants who had concerns about the pandemic. Prior to the meeting the second author contacted the participants by telephone and explained the research purpose and what their participation would entail. Participants were informed about the methods used to ensure their anonymity and data protection. All nurses contacted agreed to participate. A consent form was mailed to them and once returned signed an appointment was made at a day and time that was convenient to the participants. Consent was granted for the recording of the interview with a digital tape recorder during the physical meeting and electronically during the online meeting. The recording was deemed necessary to ensure that the researcher was focused on the interview and not on taking notes. At the beginning of the meeting the interviewer explained once more the research purpose and aims of the study. The interviews lasted 30 minutes on average.

\section{Data Analysis}

Data were analyzed based on thematic analysis $(33,35)$. The digital recordings were transcribed. The transcriptions of the recordings were read several times until the researcher (second author) familiarized herself with the utterances participants shared. In the next step, we focused on the initial coding of the material. Information from the interviews related to the research questions were grouped together. During this process several themes and subthemes emerged which were identified (Table 2, Table 3). The next step of the analysis included extension of the themes and sub-themes (or otherwise codes and sub-codes) for expanding the research team's understanding about the experiences of nurses who care for people who are mechanically ventilated and conscious. The researchers were aware that there would be both singularities as well as universalities among participants' experiences. For this reason, they paid attention on the themes and sub-themes related to the purpose and aims of the current study (36).The other two authors followed-up the process of data analysis. Regular meetings occurred between the three authors during which the trustworthiness of the data analysis was ensured (36).

Table 2

Example of the process of analysis from quote to category.

\begin{tabular}{|c|c|c|c|}
\hline Quote & Codes & $\begin{array}{l}\text { Sub- } \\
\text { categories }\end{array}$ & Categories \\
\hline $\begin{array}{l}\text { In order to communicate you have to yell because you have a mask that is thick with a filter in front of } \\
\text { you, a face-shilt which is a barrier to communication. The COVID-19 uniform is all over you and it's } \\
\text { covering your ears }\end{array}$ & $\begin{array}{l}\text { Difficult } \\
\text { communication }\end{array}$ & $\begin{array}{l}\text { Protective } \\
\text { dressing } \\
\text { equipment }\end{array}$ & $\begin{array}{l}\text { COVID-19 } \\
\text { communication } \\
\text { barriers }\end{array}$ \\
\hline
\end{tabular}

Table 3

Categories and Sub-categories of communication strategies

\begin{tabular}{|ll|}
\hline Unaided forms & Aided forms \\
\hline - Eye contact & - Pen and paper \\
- Head nodding & - Mobiles \\
- Hands or legs movements & - Frames \\
- Touching & - Boards with markers \\
- Close-ended questions & - Tablets \\
\hline
\end{tabular}

\section{Ethical considerations}

The study was conducted according to the ethical principles of medical research involving human subjects (Declaration of Helsinki, WMA, 2013) and the law for the safeguarding of personal data processing (Cyprus Republic, 138/I, 2001). The study was reviewed and approved by the National Bioethics Committee of Cyprus (Project-ID: EEBK EП 2020.01.40) and the Research Committee of the Ministry of Health. Permission to conduct the study was obtained from every hospital administrator and every ICU manager before inviting nurses to participate. All participating nurses received by post mail written and verbal information about the study and returned the informed consent signed. Additionally, written consent was obtained for the recording of the interviews (physical and online). To protect participants' privacy and anonymity we herein refer to nurses' verbatim using pseudonyms. All participants were informed of the voluntary nature of the study and the option to withdraw their consent either before, during or after the interview.

\section{Results}

\section{Communication Strategies}

The findings reveal that both patients and nurses use a variety of strategies to communicate with each other. These can be grouped as unaided and aided forms of communication. Unaided are those for which the person does not require anything external to his/her body to communicate and the opposite relates to aided forms of communication $(37,38)$.

\section{Unaided Forms of Communication}

According to participating nurses, patients use unaided forms of communication such as eye contact, head nodding, hands' or legs' movement (e.g. lift their hand upwards or grasp the hand of the nurse), pointing, lip moving, and using gestures. For example, Antria commented "when he is awake, he can communicate and collaborate with others. He can blink, grip someone's hand, make face and limb movements, let you know when he is in pain, using nodding 
and gestures". Participants commented that they encourage patients to use unaided methods of communication as ventilation acts as a barrier for verbal communication. For instance, Thalia said: "When I ask him if he can hear me, [I instruct him that] he may hold my hand tight or close his eyes [to respond]".

Other examples of unaided communication are closed-ended questions and touching. As Stratos said: "There are many things that might disturb patients hospitalized in an ICU. You may ask them questions, and your questions might be, for example: Are you in pain? Does anything bother you? Can you breathe properly?". Additionally, Angelina shared: "...quite often, we use touch as a form of communication, just to reassure them by holding their hands so that they don't feel alone".

Furthermore, in the ICU environment, nurses would use all available sources of information to assess a patient's need to communicate. Angelos spoke about the way the monitor in the ICU could be of help in the communication: "Our ICU is an open space, we can see the patient. We know that we can get up and look at him/her, we can check the monitor and the patient. So, the patient may not have to use his hands or legs since I can hear him if, I mean the respirator will make a sound when he tries to move".

In addition, nurses often attempt to improve their communication interaction with patients by seeking support by other communication partners such as relatives and/or other staff. This strategy was mainly used when trying to decipher patients' letters or gestures. As Achilleas stated: “When patients don't speak Greek or English then a relative was sought to help us understand". Similarly, Angelos said: "I would fetch another nurse and ask him if he understood what the patient was trying to say". Other times, when one nurse managed to understand what a patient meant with a specific movement or facial expression (such as pain or thirst), they made sure to communicate it to nurses in the next shift. Angelos said: "Maybe a specific nurse worked more with a specific patient and understands him better, then I ask him what is the best way to communicate with this patient. It's team-work, you learn to ask for help in a ICU, you can't work alone".

As it is explained in more detail later in this paper, the COVID-19 pandemic has added several restrictions in the effective communication between nurses and patients. However, it seems that nurses are doing their best to utilize existing strategies for communication to the maximum. Angelina said: "Now you have these masks and the hats. I think the eyes are really important to make eye contact all the time because they can't see if you're smiling anymore. They can't see if you're smiling or what your facial expression is, because you have the masks on. So, it's important to make eye contact and try to be reassuring with your eye contact".

\section{Aided Forms of Communication}

Communication can take place with the support of a variety of aided methods of communication. Participating nurses shared that the most commonly used methods are pen and paper, e-tran frames, mobiles, ipads. Antria said: "We use the pain expression scale. We had a patient with Guillain-Barre who could not move his/her face at all, so we slowly started using the letter table. A speech language therapist came and explained how to use it and the meaning of its colours. But we have rarely used it. In the last few years we use tablets, pencil and paper, not so much their mobile phones". Nurses spoke about paper and boards and how they can be of help in their efforts to communicate with patients. Angelina said: "So, you get them like a piece of paper on a clipboard. And they can write things down". Loizos added: [We had] a board with a marker, there was a glass window in front of him but we could see him and he could see us. We would write messages for him like "Everything will be ok" or draw a smiley face or write "We will make it, things like that".

\section{Barriers to Communication}

A number of barriers for successful communication were identified and the findings reveal that the COVID-19 pandemic has added additional ones to those previously reported.

\section{Patients' variables}

The patients' condition, physical, mental, emotional as well as the medication regimen impacted the communication between nurses and patients who were mechanically ventilated and awake. Focusing on the physical condition, one participant specified that patients might be difficult to communicate with due to chronic medical conditions. Constantinos commented: "We had a patient who was blind and had hearing impairments. Communicating with him was very difficult, really [difficult], since he could barely hear you and could not see you either". Muscle strength related to the medical condition was also an issue during communication. As Angelos said: "you could not really tell the numbers, because when intubated they cannot hold the board properly, they don't have the strength to do so". Additionally, cognitive impairments make communication difficult and lead to abandoning all efforts. Antreas said: "If someone has a brain injury, well ok, at the beginning you try but slowly you realize that a lot of time is required". Georgia added: "Yes, there are patients who have cognitive impairments, and those cases are generally very difficult... for me, very stressful since you don't know what the specific person can actually understand."

Regarding medications Angelina said: "Sometimes if they're on medication for pain relief, they can be a bit confused and that is an issue. Because they want to pull the tube out". Furthermore, psychological factors such as depression might deprive patients from any desire to engage in communication. As Agamemnon said: " one moment you see them being reactive, the next moment they might stop talking at all. They may be depressive, it's different for everyone". Furthermore, language can act as a barrier, albeit participants seemed to be able to find solutions. Achilleas commented: "In cases when the patients can't understand Greek or English, a relative may help us".

\section{Nurses' variables}

Some nurses mention that communication depends on nurses' personal attitudes (such as willingness and compassion). As Thalia said: "all nurses don't have the same willingness or the same compassion for the patient". Additionally, nurses identified lack of ICU experience as another barrier because even if they are willing to communicate with their patients they need to prioritize their activities, namely physical safety. As Angellina said: "sometimes especially if you're a new nurse, it's very difficult to balance the technology and understanding the medications and the pumps, but also the patient's psychological needs".

Page 5/12 
Furthermore, nurses identified work overload which leads to burn out as a barrier to communication. As Thalia said: "The main obstacle in this situation is the [subsequent] burnout because you have to support yourself, if I am exhausted, [if] I am dismayed how would I help the other person in front of me, when I myself am tired, I'm just trying to go to do my job, do the basics and leave, it may sound cold and raw, what l've just said but it does happen".

\section{ICU environment}

The participants referred to the physical environment of the ICU which occasionally acts as a barrier to communication with patients. Especially when it comes to noise, lights, lack of windows and sun, Antria commented: "Since the ICU is now an open space, patients are next to each other, there is a lot of noise, fuss, lights... He/she can't use his/her voice to tell you [something], so the quieter it is the easier it is for you to understand, that's all". Georgia added: "For patients that stay a long time in place like an ICU, it is important to see windows and sun light, because if they don't see them, they become very confused and it's a pity to stress them". In addition, nurses mention that when patients realize they are in the ICU they experience distress due to the unfamiliar environment which makes them reluctant to engage in communication. Thalia said: "First, they open their eyes and they see a foreign place with strangers around them... where they cannot understand what is happening, I definitely think that their first thought is My Lady [Mary], where am I now?". Another barrier identified related to an open ICU is the lack of privacy which can cause patients to experience negative feelings and lack of motivation for communication. Agamemnon shared: "You might be performing weaning, trying to wake up a patient, and after a while the patient may see someone else dying, which causes even more terror, so they might react in a strong way or they might even stop talking, become depressed".

\section{Assistive technology}

As mentioned above, communication interaction can take place both in unaided and aided forms. Regarding the aided forms of communication, participants commented that ICUs in Cyprus might not have such a variety of equipment to support the interaction between nurses and patients. Thalia commented: "The facilities do not have the necessary means. I believe that, even though I haven't looked into it, I'm sure that in foreign countries there are several means of communication. But in Cyprus we know nothing".

Naturally, the lack of nurses' knowledge and training on how to use assistive technology acts as a barrier to communication. As Alexandros said: " We have insufficient knowledge, that is, if you were to ask me a question, I'll tell you I don't know, I was not taught anything, that is, they gave me a tool and they presented it to me and told me that [for] this patient at this given moment you can work on him [using] these things, and it will have a good result. Namely, this is what we do, act by experience". Participants also mentioned that patients' lack of knowledge on how to use assistive technology to communicate can also ask as a barrier. Loizos commented: "There are very few patients who can use a tablet since they are a bit old and I think that it's more difficult for them".

\section{COVID-19 related communication barriers}

Undoubtedly, COVID-19 added communication barriers between nurses and patients mainly due to the compulsory use of protective health equipment. Participants mentioned that health protocols impaired their efforts to communicate imposing limitations on nurses' speech output, facial expressions and touching. Focusing on speech output Angelos shared how the nurse uniform and the mask prohibited the communication interaction that usually takes place between a nurse and a patient. As he said: "Since we had to wear a very thick mask with a filter, we had to shout so that we could be heard. But having a shield mask on the front stops the sound. Also, we had to wear a full body uniform covering even our ears. So, there were three obstacles, which inhibited the communication between the nurses, between nurses and physicians, and between nurses and patients. Having all of our senses intact helps [nurses] but don't forget that patients don't".

Regarding facial expressions, Agamemnon acknowledges the importance of facial expressions in the communication with patients and shares the barriers that COVID-19 restrictions imposed: "[the pandemia impacted everyday practice] very much, since the patient cannot see your face. This does not allow you to communicate or express your feelings of support, so that [the patient] can understand that you are really there. He/she can only see your eyes and nothing else. He/she can't see your face expressions, and this is bad for a patient who wakes up and what he/she sees is a faceless person".

Gentle touch is a communication strategy used by nurses but the findings show that due to COVID-19 restrictions this is no longer practiced. As Loizos shared: "When you know that a patient does not have something like COVID for example, the communication is easier. Meaning that you can go near the patient, talk to him/her from a short distance, touch him/her. Even with a glove on, this can calm him/her a bit. Dealing with COVID patients is very different".

Furthermore, nurses stress that the protective dressing equipment causes time limitations which combined with the work overload and staff shortages put communication efforts lower in the list of nurses' priorities. Agamemnon commented: "It is usually about personal safety measures that exist and the issue of time, because there is currently a lot of work in the ICU, there is less staff, you may have 3-4 patients, and usually you will pay less attention to the patient when he's awake, since he has fewer needs". The theme of "safety" returns when Thalia says: "In the other ICU, since we now have two, since, well you cannot be inside all the time, for your own safety". What is worse, some nurses are concerned that the protective equipment might make patients to be afraid of health care professionals. Georgia mentioned: "We don't want patients to be scared when they see us. Our appearance is scary, because you see a person standing over your head, wearing a mask, a shield, a scrub cap, gloves, a robe".

\section{Discussion}

Patients hospitalized in an ICU, who are mechanically ventilated but awake, have complex communication needs which need to be addressed in a holistic and respectful way to achieve standards consistent with ethical health and nursing care $(39,40)$. This group of patients face temporary communication disability which, if not addressed appropriately, might leave a negative imprint in their memories (29). What is worse, basic patients' needs might not be addressed because they cannot be communicated (41). Interestingly, hospital accreditation standards characterize the communication disability acquired as a result of endotracheal or tracheal intubation during critical illness as a condition requiring provider assessment and accommodation (42). 
As the International Classification of Functioning, Disability and Health (ICF) model suggests, interventions aimed to assist people with disability should consider both intrinsic factors related to the individual with complex communication needs and extrinsic factors related to the environment(29). Furthermore, the Participation Model, a framework that guides AAC assessment, delineates access and opportunity barriers as two main barriers to successful implementation of AAC. Access barriers are based on issues related to the individual who uses AAC while opportunity barriers to participation are those imposed by people in the environment of the AAC user $(26,43)$. We analyzed our data based on these premises and interpreted them accordingly.

\section{Communication strategies}

Nurses who participated in this study reported using both unaided and aided forms of communication to understand the needs of mechanically ventilated patients who are awake. The various strategies of unaided communication mentioned by participants were eye contact, head nodding, hands' or legs' movement, pointing, lip moving, and using gestures. Our findings confirm results from previous studies which demonstrate that nurses use several unaided forms of communication strategies. Specifically, eye contact between nurses and patients was found to be one of the most common unaided method of communication $(5,8,10,17,19,21)$. This is not surprising as eye-contact is a natural communication method that people use during face-to-face communication. Similarly, Karlsson et al. (2012), Tingsvik et al. (2013), Happ et al. (2011), Magnus et al. (2006) reported head nodding as a communication strategy $(5,10,18,20)$. Again, head nodding is a natural way of communication since people usually move their head to express consent or rejection of something (e.g. yes/no reply).

Close-ended questions were also reported by nurses to be of use when communicating with patients. These types of questions require a yes/no response which is easily conveyed with head nodding or the eyes. In fact, close-ended questions is a strategy reported in other studies (8-10, 18, 44). Pointing was another strategy mentioned by our participants which is also reported by Happ et al. (2011) (18). Gestures were mentioned by the nurses of this study and agrees with findings by others $(5,9,17,19)$. Finally, similar to our findings, touching has been reported previously as a communication strategy of unaided communication employed by nurses with conscious, mechanically ventilated patients $(5,8,14,18)$.

Interestingly, lip movement was used as another communication strategy by participants consistent with the existing literature (8-10, 14, 19, 21, 45). However, according to our findings the COVID-19 health protocols followed in hospitals during the pandemic prohibited the implementation of this communication strategy. Nurses were obliged to wear masks which obstructed patients from reading their lips. Using visible masks have not been reported by nurses in this study or others although they are quite popular amongst speech and language therapists. Ideally, nurses should wear visible masks, to allow patients to see their lips although masks might not always be readily available.

Nurses often attempt to improve their communication interaction with patients by seeking support by other communication partners such as relatives and/or other staff. Similarly, Bergbom et al. (1993), Karlsson et al. (2015) and Rodriquez et al. (2015) mentioned that nurses often ask help from patients' relatives $(14,16)$. Specifically, Rodriguez et al. (2015) found that nurses asked family members, when available, to read patients' handwriting (19). Alternatively, as reported by participants in our study, when one nurse managed to understand what a patient meant with a specific movement or facial expression (such as pain or thirst), they made sure to communicate it to nurses in the next shift. This is in agreement with findings by Rodriguez et al. (2015) who found that when patients could not clearly communicate their needs, nurses had to "figure out the clues" and then pass those clues on "nurse to nurse to nurse, shift to shift to shift" (19).

Furthermore, nurses employed aided methods of communication to facilitate their communication with patients who were mechanically ventilated, and awake. Similar to others, nurses in this study stated that they often use pen and paper, markers and frames $(8,9,14,17-19)$. These are considered low-tech products and are widely used in ICU settings because they are relatively inexpensive. In addition, communicating via pen and paper is familiar to most people. Naturally, easy ways of communication are selected first since augmentative and alternative communication strategies require training. However, some lowtech strategies themselves require training, such as the e-tran frame. This is potentially why it was not frequently reported by nurses in our study but was used by nurses in other studies $(8-10,14,19,44)$. Lack of exposure and training to alternative and augmentative communication methods can be facilitated by bringing together communication partners (i.e. nurses) with speech and language therapists/ AAC experts who can provide appropriate support to improve communication with ICU patients who are ventilated and awake.

\section{Barriers to Communication}

Nurses in our study identified several barriers in their efforts to communicate with patients who were mechanically ventilated and awake as summarized in Table 4. Access barriers (issues related to the individual who uses AAC) were mainly related to patients' medical condition, medication regimen as well as language. Cognitive dysfunction was identified as an important barrier in this study similar to the findings of others $(9,10,18,28)$. In addition, we found that muscle weakness made it extremely difficult to even use a pen to write on a paper or hold a board to find appropriate signals. Similar findings have been reported by Holm et al. (2017) who identified that patients' level of fatigue, muscle strength, consciousness, cognitive ability and participation in care were of great importance for communication (9). Moreover, according to nurses the patients' psychological state acts as a barrier to communication. This is consistent with findings by others $(16,19)$ who reported that many patients refused to engage in any communication due to feelings of frustration of not being able to speak. Similarly, patients' feelings of anger, anxiety and depression have been reported as a communication barriers between nurses and patients (10).

Furthermore, several opportunity barriers (those imposed by people in the environment) that deter communication with mechanically ventilated patients who are awake were acknowledged by nurses in our study. Nurses' identified their own attitudes (such as willingness and compassion) along with their lack of knowledge and training on how to use assistive technology as important barriers to communication. Our findings are in line with those of others who found that lack of patience was a defining factor for nurses' attitudes towards sedation which in turn affected communication with patients (20). Also, Mortensen et al. (2019) reported that nurses in their study acknowledged their lack of knowledge and expressed willingness to improve it (21). Most importantly, our

Page $7 / 12$ 
findings are in agreement with the Participation Model which describes that skills, knowledge and attitudes are important features of the communication partner when facilitating AAC (46). For instance, if the person does not have a good attitude towards AAC forms of communication or the person lacks relevant experience then communication breakdowns will occur. Importantly, previous work showed significant improvements in nurse-patient communication in the ICU with training and the use of AAC (47). This lends serious support for the use of structured approaches to address issues related to AAC use for the improvement of communication with mechanically ventilated patients who are awake in an ICU.

Additionally, the ICU environment imposed serious challenges in the quality of the communication between nurses and awake, mechanically ventilated patients. Nurses in our study agree with others $(48,49)$ that constant noise, lack of privacy as well as lack of physical light make nurses' and patients' orientation towards communication extremely difficult. Furthermore, we found that assistive technology for aided forms of communication is not readily available in ICUs in Cyprus imposing further limitations to patients when trying to express their needs. To make matters worse, patients themselves often lack the required knowledge on how to use assistive technology which combined with ICU nurses' feelings of exhaustion due to work overload leads to communication breakdowns.

\section{COVID-19 health protocols}

Undoubtedly, the COVID-19 restrictions added communication barriers between nurses and patients mainly due to the compulsory use of protective health equipment. The latter prohibited the use of unaided communication strategies. Participants mentioned that health protocols imposed limitations on the way they talk (speech output), use facial expressions and touching to communicate with patients. Nurses in our study recognize the importance of all three strategies for successful communication with patients and elaborate on the difficulties that the pandemic is imposing on their practice. This is one of the first studies to portray the challenges that the pandemic has brought in the communication between nurses and patients who are ventilated and awake. What is worse, nurses reported that without the unaided methods of communication as basic tools (speech, face, touch), not only does communication in the ICU fail, it also causes feelings of distress to both patients and nurses.

Furthermore, the findings show that the COVID-19 pandemic has exacerbated the problem of limited time devoted to communication with mechanically ventilated patients who are awake. Acute patients' needs force nurses to prioritize their activities even if they are willing to try to communicate. Reduced staffing is a well-known problem in ICUs as it associates with reduced patient outcomes (50) but during the pandemic this problem reached unprecedented levels (51). This combined with safety considerations further exacerbated communication deficits with patients. What the consequences of these impairments in communication might be for patients is still too early to observe. Patients might take some time to be able to talk about their experience in the ICU during the pandemic which, in turn, makes it hard to understand how this experience affected their quality of life during and after ICU stay. Additionally, the impact of communication breakdowns with patients on nurses' feelings will be worth exploring in the future.

Table 4

Categories and Sub-categories of communication barriers

\begin{tabular}{|c|c|c|c|c|}
\hline Patient variables & Nurses variables & $\begin{array}{l}\text { ICU } \\
\text { characteristics }\end{array}$ & Assistive technology & COVID 19 communication barriers \\
\hline \multirow{2}{*}{$\begin{array}{l}\text {-Physical } \\
\text { condition }\end{array}$} & -Attitudes & $\begin{array}{l}\text {-Noise and } \\
\text { lights }\end{array}$ & -Lack of equipment & -Protective dressing equipment \\
\hline & $\begin{array}{l}\text {-Lack of knowledge and } \\
\text { training on AAC }\end{array}$ & \multirow{2}{*}{$\begin{array}{l}\text {-Lack of } \\
\text { privacy }\end{array}$} & \multirow[t]{3}{*}{$\begin{array}{l}\text {-Lack of patients' knowledge to use } \\
\text { assistive technology }\end{array}$} & $\begin{array}{l}\text {-Problems with unaided } \\
\text { communication }\end{array}$ \\
\hline $\begin{array}{l}\text {-Mental } \\
\text { condition }\end{array}$ & -Lack of ICU experience & & & -Limited time in patients' room due to \\
\hline $\begin{array}{l}\text {-Psychological } \\
\text { condition }\end{array}$ & & $\begin{array}{l}\text {-Foreign } \\
\text { environment }\end{array}$ & & safety protocols \\
\hline \multicolumn{2}{|l|}{-Medications } & $\begin{array}{l}\text {-Time } \\
\text { limitations }\end{array}$ & & \\
\hline -Language & & $\begin{array}{l}\text {-Staff } \\
\text { shortage }\end{array}$ & & \\
\hline
\end{tabular}

\section{Strengths and limitations}

Research on the experiences of nurses communicating with awake ventilated patients is limited. For this reason, we employed a qualitative research approach to investigate experiences of nurses working in ICUs in Cyprus. The in-depth data collection led to interesting findings regarding the experiences of nurses when trying to communicate with ventilated, awake patients during a pandemic. In terms of trustworthiness, the research team followed the guidelines proposed by Shenton et al. (2004) (52). Additionally, during the data analysis all transcripts were analysed in their original language. Following Marshall and Rossman's (2011) guidelines, transcripts were translated in English by one of the authors. The other two authors read both the original extracts and those translated from Greek to English to confirm that the translation supported the trustworthiness of the data (53). Furthermore, the composition of our research team includes academic, clinical nurses as well as speech and language therapists which helps approach patients' complex communication needs from different perspectives.

The sample was relatively homogeneous, with Cypriot-speaking nurses from three urban areas. Participants worked in various types of ICUs in Cyprus (i.e. public versus private, open versus closed) which adds to the variety of experiences that nurses might have had and covered a wide range with both short and long professional experience. Strangely, the gender distribution strangely favoured male nurses' selection which might relate to the fact that female nurses were more reluctant to participate during a pandemic due to burn-out or safety considerations. The interviewer's experience of working in intensive care facilitated a trustful relationship with the participants but could also be seen as a limitation if the participants' pre-understanding of the context was taken for granted. The interviewer was aware of this risk and strived to be open minded during the interviews, pose clarifying questions, and avoid drawing conclusions 
without reiterating participants' answers. Finally, an important strength of this study and its primary contribution is the fact that data was collected during the period of the COVID-19 pandemic. This is one of the first studies, if not the first, that explores communication barriers caused by the COVID-19 pandemic. As such, its findings can create new research areas worth of further exploration in terms of the consequences of COVID-19 to both nurses and patients.

\section{Recommendations for clinical practice}

The present study has provided an in-depth view of how nurses working in critical care communicate with patients who are mechanically ventilated but awake. The results point to the active role that nurses take in assisting patients to communicate their needs using various aided and unaided methods. Nurses need advanced communication skills given that patients who become suddenly voiceless due to intubation regard high-tech AAC devices as a useful, reliable, and acceptable alternative communication choice in the ICU (54). Correspondingly, this research needs to inform undergraduate nursing programmes in order to equip future professionals with the skills required to efficiently communicate with mechanically ventilated patients who are awake.

Alternatively, close collaboration with speech and language therapists can be achieved if they are invited in the ICU for the assessment and management of a patient with complex communication needs. This would set the stage for interdisciplinary collaboration during which speech and language therapists can share with nurses (and caregivers) their expertise on unaided methods of communication (such as visible masks) and aided tools and help them familiarize with their use to achieve efficient communication without barriers. Currently, similar interventions are being developed that employ a multi-component bundle (55). It remains to be seen how they will be received by nurses and patients. With this study, we aspire to contribute to this effort and improve the communication between nurses and mechanically ventilated, conscious patients.

\section{Conclusions}

Maintaining communicative competence (56) in an ICU environment is challenging due to the life-threatening medical conditions that usually lead to critical care. Fear of the unknown and lack of contact with caregivers can make patients who are awake while mechanically ventilated experience feelings of isolation and anxiety. Thus, it is critical to find ways to address the temporary disability using all available methods. Nurses play a crucial role as facilitators of communication and we believe that our results attest to their determination to select any available strategies to sustain communication with patients even during extremely difficult circumstances. Communication with the patient is the bases of nursing care and as noted by many participants, making eye contact became even more important during the pandemic because smiling or facial expression were not available with the masks on.

Additionally, our finding could provide a rationale for studies evaluating the impact of targeted interventions to improve nurses' knowledge and skills taking care of this vulnerable group of patients. In this context, research that contributes to our understanding of the challenges that nurses face is indispensable. Finally, the composition of research teams needs to be multidisciplinary since this will guarantee that patients complex communication needs will be approached from various angles. Hopefully, our study will contribute to future research that will include augmentative and alternative communication (AAC) methods as well as multidisciplinary research teams to assist mechanically ventilated patients who are conscious but unable to speak due to the presence of an endotracheal tube. Particularly for Cyprus and other Mediterranean countries, where no previous research was found, our study aspires to foster collaboration between multinational research teams and national nursing bodies to improve education and guidelines for the communication between nurses and conscious, ventilated patients.

\section{Declarations}

\section{Acknowledgements}

The authors wish to thank all the nurses who participated in this study for their time and invaluable comments.

\section{Authors' information}

MK: Assistant Professor, Cyprus University of Technology, Faculty of Health Sciences, Department of Nursing.

CC: Registered Nurse, American Medical Center/American Heart Institute, Nicosia, Cyprus.

EP: Lecturer, Cyprus University of Technology, Faculty of Health Sciences, Department of Rehabilitation Sciences.

\section{Authors' Contributions}

MK and EP conceived and designed the study. CC collected the data. Analysis of the data was performed by EP and CC. MK and EP wrote the manuscript and CC made substantial contributions to specific sections. All authors read and approved the final manuscript.

\section{Funding}

Funding through the "CUT Open Access Author Fund" (Cyprus University of Technology Library) was secured to cover the expenses for open access publication.

\section{Availability of data and materials}

The dataset supporting the conclusions is available from the corresponding author on reasonable request. 


\section{Declarations}

\section{- Ethics approval and consent to participate}

Ethics approval was given by the Cyprus National Bioethics Committee (EEBK EП 2018.01.154) and by the Research Committee of the Ministry of Health. Prior to data collection, ethical approval was obtained from the Institutional Review Board of each hospital where participants work. All nurses were fully informed about the study and informed consent was obtained from all subjects. During data collection, all methods were performed in accordance with the relevant guidelines and regulations for the treatment of human subjects (Declaration of Helsinki). The anonymity and confidentiality of the participants were assured and data were treated as strictly confidential.

\section{- Consent for publication}

Not applicable.

\section{- Competing interests}

The authors declare that they have no financial or non-financial competing interests.

\section{References}

1. Puntillo KA, Max A, Timsit JF, Vignoud L, Chanques G, Robleda G, et al. Determinants of procedural pain intensity in the intensive care unit: The Europain ${ }^{8}$ study. Am J Respir Crit Care Med. 2014 Jan;189(1):39-47.

2. Devlin JW, Skrobik Y, Gélinas C, Needham DM, Slooter AJC, Pandharipande PP, et al. Clinical Practice Guidelines for the Prevention and Management of Pain, Agitation/Sedation, Delirium, Immobility, and Sleep Disruption in Adult Patients in the ICU. Crit Care Med. 2018 Sep;46(9):e825-73.

3. Stephens RJ, Dettmer MR, Roberts BW, Ablordeppey E, Fowler SA, Kollef MH. Practice patterns and outcomes associated with early sedation depth in mechanically ventilated patients: a systematic review and meta-analysis. Crit Care Med. 2018;176(3):139-48.

4. Hughes CG, Girard TD, Pandharipande PP. Daily Sedation Interruption Versus Targeted Light Sedation Strategies in ICU Patients. Crit Care Med. 2013 Sep;41(9 SUPPL.1):39-45.

5. Karlsson V, Forsberg A, Bergbom I. Communication when patients are conscious during respirator treatment-A hermeneutic observation study. Intensive Crit Care Nurs [Internet]. 2012;28(4):197-207. Available from: http://dx.doi.org/10.1016/j.iccn.2011.12.007.

6. Khalaila BR, Zbidat W, Anwar K, Bayya A, Linton DM, Sviri S. Communication difficulties and psychoemotional distress in patients receiving mechanical ventilation. Am J Crit Care. 2011;20(6):470-9

7. Samuelson KAM, Lundberg D, Fridlund B. Stressful memories and psychological distress in adult mechanically ventilated intensive care patients - A 2month follow-up study. Acta Anaesthesiol Scand. 2007;51(6):671-8.

8. Leathart AJ. Communication and socialisation (1): an exploratory study and explanation for nurse- patient communication in an ITU. 1994;(February):93104.

9. Holm A, Dreyer P. Nurse-patient communication within the context of non-sedated mechanical ventilation: A hermeneutic-phenomenological study. Nurs Crit Care. 2017;23(2):88-94.

10. Magnus VS, Turkington L. Communication interaction in ICU-Patient and staff experiences and perceptions. Intensive Crit Care Nurs. 2006;22(3):167-80.

11. Campbell GB, Happ MB. Symptom identification in the chronically critically ill. AACN Adv Crit Care. 2010;21(1):64-79.

12. Handberg C, Voss AK. Implementing augmentative and alternative communication in critical care settings: Perspectives of healthcare professionals. $J$ Clin Nurs. 2018;27(1-2):102-14.

13. UN General Assembly. Universal Declaration of Human Rights | United Nations. General Assembly resolution. 1948. p. 217.

14. Bergbom-Engberg I, Haljamăe H. The communication process with ventilator patients in the ICU as perceived by the nursing staff. Intensive Crit Care Nurs [Internet]. 1993 Mar 1 [cited 2019 May 13];9(1):40-7. Available from: https://www.sciencedirect.com/science/article/pii/096433979390008L? via\%3Dihub.

15. Laerkner E, Egerod I, Ploug H. Nurses ' experiences of caring for critically ill, non-sedated, mechanically ventilated patients in the Intensive Care Unit : A qualitative study. Intensive Crit Care Nurs [Internet]. 2015;31(4):196-204. Available from: http://dx.doi.org/10.1016/j.iccn.2015.01.005.

16. Veronika K, Ingegerd B. ICU Professionals' Experiences of Caring for Conscious Patients Receiving MVT. West J Nurs Res. 2015;37(3):360-75.

17. IJssennagger CE, Ten Hoorn S, Van Wijk A, Van den Broek JM, Girbes AR, Tuinman PR. Caregivers' perceptions towards communication with mechanically ventilated patients: The results of a multicenter survey. J Crit Care [Internet]. 2018;48:263-8. Available from: https://doi.org/10.1016/j.jcrc.2018.08.036.

18. Happ MB, Garrett K, Thomas DDV, Tate J, George E, Houze M, et al. Nurse-patient communication interactions in the intensive care unit. Am J Crit Care. 2011;20(2):28-40.

19. Rodriguez CS, Spring HJ, Rowe M. Nurses ' Experiences of Communicating With Hospitalized, Suddenly Speechless Patients. 2015.

20. Tingsvik C, Bexell E, Andersson AC, Henricson M. Meeting the challenge: ICU-nurses' experiences of lightly sedated patients. Aust Crit Care [Internet]. 2013;26(3):124-9. Available from: http://dx.doi.org/10.1016/j.aucc.2012.12.005.

21. Mortensen CB, Kjær MBN, Egerod I. Caring for non-sedated mechanically ventilated patients in ICU: A qualitative study comparing perspectives of expert and competent nurses. Intensive Crit Care Nurs. 2019;52:35-41. 
22. Löf L, Berggren L, Ahlström G. ICU patients' recall of emotional reactions in the trajectory from falling critically ill to hospital discharge: Follow-ups after 3 and 12 months. Intensive Crit Care Nurs. 2008 Apr;24(2):108-21.

23. Peplau HE. Interpersonal Relations In Nursing:A conceptual Frame of Reference for Psychodynamic Nursing. New York: G. P. Putnam's Sons; 1952.

24. Berlo DK. The process of communication; an introduction to theory and practice. New York: Rinehart and Winston; 1960.

25. Happ MB, Seaman JB, Nilsen ML, Sciulli A, Tate JA, Saul M, et al. The number of mechanically ventilated ICU patients meeting communication criteria. Hear Lung J Acute Crit Care [Internet]. 2015 Jan 1 [cited 2021 Jun 17];44(1):45-9. Available from: https://pubmed.ncbi.nlm.nih.gov/25261939/.

26. Beukelman DR. Augmentative and alternative communication: supporting children and adults with complex communication needs / by David R. Beukelman and Pat Mirenda [Internet]. Augmentative \& alternative communication. 2013 [cited 2021 Jun 17]. Available from: https://books.google.com.cy/books/about/Augmentative_and_Alternative_Communicati.html?id=oUQZMAEACAAJ\&redir_esc=y.

27. Finke EH, Light J, Kitko L. A systematic review of the effectiveness of nurse communication with patients with complex communication needs with a focus on the use of augmentative and alternative communication. J Clin Nurs. 2008;17(16):2102-15.

28. Radtke JV, Baumann BM, Garrett KL, Happ MB. Listening to the voiceless patient: Case reports in assisted communication in the intensive care Unit. J Palliat Med [Internet]. 2011 Jun 1 [cited 2021 Jun 18];14(6):791-5. Available from: /pmc/articles/PMC3107580/.

29. World Health Organization. International classification of functioning, disability and health (ICF). 2013.

30. Raghavendra P, Bornman J, Granlund M, Björck-Äkesson E. The World Health Organization's International Classiffication of Functioning, Disability and Health: Implications for clinical and research practice in the field of augmentative and alternative communication. AAC Augment Altern Commun. 2007 Dec;23(4):349-61.

31. Polit DF. Nursing research: generating and assessing evidence for nursing practice. Wolters Kluwer Health; 2017. 488 p.

32. O'Brien BC, Harris IB, Beckman TJ, Reed DA, Cook DA. Standards for reporting qualitative research: A synthesis of recommendations. Acad Med [Internet]. 2014 [cited 2021 Jun 17];89(9):1245-51. Available from: https://pubmed.ncbi.nlm.nih.gov/24979285/.

33. Patton M. Qualitative Research \& Evaluation Methods 3rd ed. [Internet]. Vol. 3. 2002 [cited 2021 Jun 17]. 3-4 p. Available from: https://books.google.com.cy/books/about/Qualitative_Research_Evaluation_Methods.html?id=FjBw2oi8El4C\&redir_esc=y.

34. Dworkin SL. Sample size policy for qualitative studies using in-depth interviews [Internet]. Vol. 41, Archives of Sexual Behavior. Springer Science and Business Media, LLC; 2012 [cited 2021 Jun 17]. p. 1319-20. Available from: https://pubmed.ncbi.nlm.nih.gov/22968493/.

35. Miles MB, Huberman AM. Qualitative analysis: an expanded sourcebook, 2nd edition [Internet]. 1994 [cited 2021 Jun 17]. 354 p. Available from: https://books.google.com.cy/books/about/Qualitative_Data_Analysis.html?id=U4IU_-wJ5QEC\&redir_esc=y.

36. Elo S, Kääriäinen M, Kanste O, Pölkki T, Utriainen K, Kyngäs H. Qualitative Content Analysis. SAGE Open [Internet]. 2014 Jan 1 [cited 2021 Jun 17];4(1):215824401452263. Available from: https://journals.sagepub.com/doi/10.1177/2158244014522633.

37. Lloyd LL, Fuller DR. Toward an Augmentative and Alternative Communication symbol taxonomy: A proposed superordinate classification. Augment Altern Commun [Internet]. 1986;2(4):165-71. [cited 2021 Jun 17 ;.. Available from: /record/1988-16435-001.

38. Pampoulou E, Fuller DR. Exploring AAC graphic symbol choices: a preliminary study. J Enabling Technol. 2020 Dec 7;14(3):171-85.

39. American Medical Association. Ethical Force Program Consensus Report. Improving Communication-Improving Care. Chicago, IIl; 2006.

40. American Nurses Association. Code of Ethics for Nurses With Interpretive Statements. Silver Spring, Md; 2001.

41. Gustafsson N, Leino-Kilpi H, Prga I, Suhonen R, Stolt M. Missed care from the patient's perspective - a scoping review [Internet]. Vol. 14, Patient Preference and Adherence. Dove Medical Press Ltd.; 2020 [cited 2021 Jun 17]. p. 383-400. Available from: /pmc/articles/PMC7049852/.

42. The Joint Commission. New and Revised Standards and EPs for Patient-Center Communication-Hospital Accreditation Program. 2010.

43. Beukelman DR, Mirenda P. Augmentative and alternative communication: Supporting children and adults with complex communication needs 4th ed. 4th ed. Baltimore: Paul H Brookes; 2005.

44. Alasad J, Ahmad M. Communication with critically ill patients. J Adv Nurs [Internet]. 2005 May 1 [cited 2019 May 13];50(4):356-62. Available from: http://doi.wiley.com/10.1111/j.1365-2648.2005.03400.x.

45. Bekker C, Nørregaard MB, Egerod I. Intensive \& Critical Care Nursing Caring for non-sedated mechanically ventilated patients in ICU: A qualitative study comparing perspectives of expert and competent nurses. 2019;52:35-41.

46. Theodorou E, Pampoulou E. Investigating the Assessment Procedures for Children With Complex Communication Needs. Commun Disord Q [Internet]. 2020 Sep 30 [cited 2021 Jun 18]; Available from: https://doi.org/10.1177/1525740120960643.

47. Happ MB, Garrett KL, Tate JA, DiVirgilio D, Houze MP, Demirci JR, et al. Effect of a multi-level intervention on nurse-patient communication in the intensive care unit: Results of the SPEACS trial. Hear Lung J Acute Crit Care [Internet]. 2014 Mar [cited 2021 Jun 17];43(2):89-98. Available from: https://pubmed.ncbi.nlm.nih.gov/24495519/.

48. Bion V, Lowe ASW, Puthucheary Z, Montgomery H. Reducing sound and light exposure to improve sleep on the adult intensive care unit: An inclusive narrative review [Internet]. Vol. 19, Journal of the Intensive Care Society. SAGE Publications Inc.; 2018 [cited 2021 Jun 17]. p. 138-46. Available from: https://pubmed.ncbi.nlm.nih.gov/29796071/.

49. Simons KS, Van Den Boogaard M, De Jager CPC. Impact of intensive care unit light and noise exposure on critically ill patients. Vol. 27: Netherlands Journal of Critical Care NETH J CRIT CARE; 2019.

50. Numata Y, Schulzer M, Van Der Wal R, Globerman J, Semeniuk P, Balka E, et al. Nurse staffing levels and hospital mortality in critical care settings: Literature review and meta-analysis [Internet]. Vol. 55, Journal of Advanced Nursing. J Adv Nurs; 2006 [cited 2021 Jun 17]. p. 435-48. Available from: https://pubmed.ncbi.nlm.nih.gov/16866839/. 
51. Raurell-Torredà M. Management of ICU nursing teams during the COVID-19 pandemic. Enfermería Intensiva (English ed) [Internet]. 2020 Apr 1 [cited 2021 Jun 18];31(2):49-51. Available from: https://www.elsevier.es/en-revista-enfermeria-intensiva-english-edition-430-articulo-management-icu-nursing-teamsduring-S2529984020300197.

52. Shenton AK. Strategies for ensuring trustworthiness in qualitative research projects. Educ Inf. 2004;22(2):63-75.

53. Marshall C, Rossman GB. Designing Qualitative Research. 5th ed. [Internet]. 2011 [cited 2021 Jun 17]. Available from: https://books.google.com.cy/books? id=RbqXGjKHALoC\&pg=PA291\&lpg=PA291\&dq=Marshall,+C.+and+Rossman,+G.,+2006.+Designing+qualitative+research.+4th+ed.\&source=bl\&ots=BOfEy

54. Ju XX, Yang J, Liu XX. A systematic review on voiceless patients' willingness to adopt high-technology augmentative and alternative communication in intensive care units. Intensive Crit Care Nurs [Internet]. 2021;63:102948. Available from: https://doi.org/10.1016/j.iccn.2020.102948.

55. Holm A, Karlsson V, Nikolajsen L, Dreyer P. Strengthening and supporting nurses' communication with mechanically ventilated patients in the intensive care unit: Development of a communication intervention. Int J Nurs Stud Adv [Internet]. 2021;3(March):100025. Available from: https://doi.org/10.1016/j.jinsa.2021.100025.

56. Light J, McNaughton D. Communicative competence for individuals who require augmentative and alternative communication: A new definition for a new era of communication? [Internet]. Vol. 30, AAC: Augmentative and Alternative Communication. Taylor \& Francis; 2014 [cited 2021 Jun 17]. p. 1-18. Available from: https://www.tandfonline.com/action/journallnformation?journalCode=iaac20. 